\title{
Planning for Pregnancy - what do men and women of child-bearing age think about health status before trying to conceive?
}

\author{
L. McGowan, B. Cairns and J. Woodside \\ Centre for Public Health, Queen's University Belfast, BT12 6BA, UK.
}

Preconception (pre-pregnancy) health and lifestyle status of men and women is important for the success of a pregnancy with regards to conception, gestation and maternal and child outcomes ${ }^{(1)}$. Preconception care is defined by the WHO as, "Medical, behavioural and social health interventions to women and couples before conception occurs. . to improve maternal and child health...short and long term"(2). Evidence mounts for the importance of preconception lifestyle factors such as diet, weight, alcohol, smoking etc., yet studies indicate that few women and/or couples plan for pregnancy; with approximately $48 \%$ of pregnancies in Western Europe unintended ${ }^{(3)}$. Pregnancy timing is important, as a failure to plan when you fall pregnant is associated with poorer and higherrisk health and lifestyle behaviours, as there is little time for any positive intervention ${ }^{(4)}$. This is reflected in statistics showing more than one in two women are considered to be overweight/obese at 12 weeks gestation in Northern Ireland ${ }^{(5)}$.

An online, cross-sectional, quantitative survey was developed to assess the perceptions of men and women of child-bearing age regarding preconception care across a range of health behaviours and gauge adherence to current diet and lifestyle advice. Questions were based on validated measures where possible and new measures - informed by the literature - were subject to extensive personal and public involvement (PPI) and pilot-testing. Data was analysed via descriptives, chi-squared tests and graphical comparisons using (IBM) SPSS Version 25. Ethical approval was granted from QUB Research Ethics Committee.

Four hundred and twenty four participants between the ages of 18-60 years with and without children participated, with 333 full completions $(73.3 \%)$. Of the respondents, $86.1 \%$ were female and the average age was 28.7 years (SD 9.4). Most respondents resided in Northern Ireland (71.6\%), 50.7\% were aged 18-25 and the average body mass index (BMI) was $24 \cdot 8 \mathrm{~kg} / \mathrm{m}^{2}$ (SD $4 \cdot 7$ ). In most cases, female respondents were more agreeable to the importance of preconception health behaviours for both men and women to engage in (i.e., greater numbers agreed health practices such as consuming a healthy diet, being a healthy weight, vitamin/supplements use etc. were important). However, more men agreed/strongly agreed regarding the importance of a medical consultation (e.g. seeing a GP) prior to trying for a baby for both men and women (55.5\% and 73.4\%, respectively) than women did for both men and women (39.7\% and $52.4 \%$, respectively). Stopping smoking/alcohol and engaging in regular exercise appeared to be the most important preconception health practices (rated by both males and females). Diet quality as measured by the Eating Choices Index was relatively high overall (13.3 SD 2.7), with no significant differences based on age or sex $(p>0.05)$.

In conclusion, there is considerable variability between males and females regarding their views on preconception health behaviours, and upon those behaviours which are most important for each sex to engage in respectively. The survey targeted adults of child-bearing age and most adults were approaching overweight status, although diet quality was reasonable. More needs to be done in terms of highlighting the impact of preconception health and lifestyle behaviours on fertility rates, pregnancy-, maternaland child-health outcomes.

1. Scott-Pillai R, Spence D, Cardwell CR et al. (2013) BJOG 120, 932-939.

2. World Health Organisation (2013) Preconception care http://apps.who.int/iris/bitstream/handle/10665/78067/9789241505000_eng.pdf;jsessionid= D78D1985730F4244EF499862436F5884?sequence=1 (accessed March 2018).

3. Sedgh G, Singh S, Hussain R (2014) Stud Fam Plann 45, 301-314.

4. Cheng D, Schwarz EB, Douglas E et al. (2009) Contraception 79, 194-198.

5. Children's Health in Northern Ireland (2017) Public Health Agency for Northern Ireland, Public Health Intelligence Unit. 